\title{
Effects of Multicomponent Exercise Prescription on Cognitive and Functional Fitness for Frailty Syndrome
}

\author{
Pin-Yu, Wu*, Lee-Lan Cheng , \\ Ph. D. Student, Department of Adult and Continuing Education, National Chung-Cheng University, Chiayi, \\ Taiwan
}

*Corresponding Author: Pin-Yu, Wu, Ph. D. Student, Department of Adult and Continuing Education, National Chung-Cheng University, Chiayi, Taiwan

\begin{abstract}
:
Purpose: The purpose of this study was to investigate the effects of a multicomponent exercise on cognitive and functional fitness for Frailty Syndrome.

Methods: This study applied a pre-experimental design and conducted a one-group pretest-posttest experimental design. Participants were 20 volunteer older adults with a frailty syndrome (above 65 years old) enrolled in multicomponent exercise prescription program at senior center. The study was implemented during a 12-week period. The pretest was administered during the first week. A multicomponent exercise core program for frail elder's training in this study was adopted from American College of Sports Medicine (ACSM). The core program principles and training method were included: Warm-up and Cool-down, Flexibility training, Resistance training, Aerobic endurance training, Balance and mobility training. Participants completed a multicomponent physical fitness program, the effects on cognitive and functional fitness of learning outcome were examined across posttest.
\end{abstract}

Results: The results revealed that the model of multidimensional exercise program on performance program is significant effects for frailty. The results of this study lend support to others who indicate that exercise prescription positively affects frail adults' cognitive and functional fitness.

Conclusion: The result of this study indicated that the positive effects of the 12-week multicomponent training course improved the cognitive and functional fitness. The implications for effects of multicomponent fitness prescription on task performance program to promote frail adults on cognitive and functional fitness and future research are discussed.

Key words: Multicomponent Fitness, Frailty Syndrome, Cognitive, Functional Fitness

\section{INTRODUCTION}

In recent years, the issue of frailty syndrome is becoming increasingly important as the population of older adults grows (Zhang, N, Zhu, W. L., Chen, W., 2019; Jackson, Sapey, Lord, 2017).Frailty in older adults refers to the loss of some functional abilities(Fried, Tangen, Walston, et al.,2001). However, frailty is not synonymous with disability. Frailty can be seen as the risk of instability and loss of function as brought about by the functional degradation of various body systems(Angulo, El Assar , Rodríguez-Mañas,2016; Ruan, Yu, Chen, Bao, \&He,2015). Frailty can also be seen as a biological syndrome that includes a reduced level of resistance in the face of multiple sources of pressure due to a decline in physiological and psychological functions (Keevil, Romero-Ortuno,2015). Frailty is a geriatric syndrome that affects multiple domains of human functioning. A variety of problems contributes to the development of this syndrome; three key components includingsarcopenia, cognitive impairment, such as Alzheimer disease, and poor nutritional status were an important determinant of this condition (Heath, \& Stuart, 2002; Laura Lorenzo-López, et al., 2016; Sayer, et al, 2008). Therefore, many forms of frailty exist, including functional, medical, intellectual and psychological, and physiological frailty.

Considering that older adults form the population's fastest-growing age group, and also that a considerable proportion of this group will be frail individuals in the future, it can thus be inferred that the frailty problem will grow in severity going forward (Huang, \& Wang, 2018; Wojtek, \&ChodzkoZajko, 2015). Frailty has been described as a dominant characteristic that can be observed via five 
dimensions, namely, unintentional weight loss, self-reported exhaustion, low levels of physical activity, slowness, and weakness. An individual who exhibits three or more of these five symptoms may be considered to be suffering from frailty. Using this definition, about $7 \%$ of older adults aged 65 years and above meet the criteria for this definition (Bortz, 2002; Fried, Tangen, Walston, et al.,2001; Heath, \& Stuart, 2002). A frail adult often has to deal with one or more disabilities (such as auditory and visual impairments) which can affect his or her ability to perform the activities of daily living. However, exercise and physical activities can improve the physical functions of older adults and thereby help to prevent and/or reduce their frailty (Lorenzo-López, 2017 ).

The effects of physical fitnesswere one of the key reasons that affect health. Lack of physical activity will increase mortality and morbidity of elderly (Perracini, Franco, Ricci, \& Blake, 2017).Many studies have examined the benefits of exercise in frail elders living in skilled nursing facilities, where it is convenient to conduct and assess the effect of group-centered interventions(Wojtek, \&ChodzkoZajko, 2015 ). These institutionalized populations, who often have varying degrees of cognitive impairment, can be challenging both when trying to establish motivation for exercise and when creating opportunities for group reinforcement and participation. Study variables on which exercise interventions have had an impact include those directly related to the exercise itself changes in mobility or strength.

The development of effective model on prescribing exercise that seek to optimize the processing of new learning strategies is an essential issue of for frail elder instruction. Traditional instructional design to exercise programming for frail elders have generally focused on single training for improving functional physical fitness. Although these goals may be appropriate for some benefits for older adults, alternative goals may be helpful to accommodate the needs of older adults with more diverse levels of health combined with cognitive function, there are few research-based guidelines delving into prescribing exercise which enhance on depth of effective multicomponent exerciseon learning outcome. This study aimed to investigate the effects of a multicomponent exercise on cognitive and functional fitness among the elderly on frailty.

\section{METHODS}

\subsection{Participants}

Participants in this study were recruited from our volunteer databases, which included elderly individuals (65 years and over). The inclusion criteria we used were dwelling in the community and being 65 years or age or older. 25 prospective participants with a Clinical Frailty Rating (Fried, et. al., 2001)including 20 pre-frail and 5frailenrolled in a multicomponent prescription program. For random reasons (illness, 30 percentage absent, not finished test) were not available for 5 subjects. The final analysis was performed on a sample of 20 volunteer participants (age: 79.77 \pm 7.06 , height: 154.9 \pm 9.3 、 weight: $55.5 \pm 7.25$.

\subsection{Intervention}

Participants in the multicomponent exercise group exercised under the supervision of senior physical education professor and physiotherapists for $90 \mathrm{~min} / \mathrm{d}, 2 \mathrm{~d} / \mathrm{wk}$, during the 12 weeks. Two senior physical education professor and one physiotherapists involved in geriatric rehabilitation and three well-trained instructors conducted each intervention. The exercise prescriptions for frail older adults should be designed to suit individual abilities and preferences. The three primary objectives of exercise prescriptions for frail older adults are as follows: increased muscle strength, increased cardiovascular endurance, and increased overall physical activity and energy expenditure (ACSM, 2016; Wojtek, \&Chodzko-Zajko, 2015).

A multicomponent exercise core program for frail elder's training in this study was adopted fromAmerican College of Sports Medicine. (ACSMS, 2016) and Physical activity instruction of older adults (Jones \& Rose, 2005). The core program principles and training method were included: Warmup and Cool-down, Flexibility training, Resistance training, Aerobic endurance training, Balance and mobility training.It would also be helpful if an individual's activities of daily living and his or balance can be improved via his or her involvement other exercise programs. For the cognitive training factor, the design of the intelligent board hardware, a group exercise intervention tool, included a $4 \mathrm{M} * 1.8 \mathrm{M}$ artificial grass field for physical activities. The various shapes, colors, lines, and numbers on the 
surface corresponded to cognitive science functions and conformed with requirements regarding older adults' safety, tactile sensations, interactions, and ergonomics (Huang, Wang, \& Lin, 2016).

For the risk management and issues, the physiotherapists and well-trained instructors provided ongoing safety monitoring to prevent adverse accidents such as falling during the program.It is especially important to avoid exhaustion, which is a negative reinforcement to exercise(ACSM, 2016). After an exercise regimen has been incorporated into the patient's routine, heart rate monitoring can provide the patient and involved care givers feedback about exercise intensity.

In the absence of cardiac or respiratory symptoms, a maximum heart rate of $60 \%$ to $75 \%$ of the predicted maximum heart rate should be set as a ceiling. In this study, the training program for frail adults was adopted recent guidelines suggest that for patients planning low-intensity exercise, heart rates remain below $60 \%$ of the predicted maximal rate ( 220 beats per minute minus the patient's age), the physician can use clinical judgment to recommend an exercise stress test (ACSM, 2016; Wojtek, \&Chodzko-Zajko, 2015). For the well design for training program, daily home-based exercise in addition to structured program and outdoor walking was recommended to the participants.

\subsection{Data Collection and Analysis}

Data analysis were applied to each of the dependent variables. A t-test was use for pre -post one group experimental design. After participants completed 10 weeks of training program, a post-test was delivered. The material as measurement on cognitive function for participations was a Mini Mental State Examination, MMSE (Folstein, Folstein, \& McHugh, 1975). The MMSE is also recommended by the National Institute for Health and Clinical Excellence (NICE) as a standard tool for dementia screening. The MMSE score indicates the severity of dementia, and the maximum score is 30 points.

The scale consists of 11 items and 30 answers, with 1 point given for each correct answer. A score of 24-30 points indicates intact cognitive functions; a score of 18-23 points indicates MCI; a score of 017 points indicates severe cognitive impairment. The 11 items of the scale include five constructs, namely, orientation, registration, attention and calculation, recall, and language. Their content examines conceptions of current time and place, the repetition of words, simple mathematical calculations, and language use and understanding. The MMSE also evaluates visual-construction skills (Folsteing et al., 1975). The sample inclusion criteria in this study included an MMSE score of 18-23 points indicating MCI.

In addition, the function fitness evaluation was adopted from Senior Fitness Test, SFT (Rikli\& Jones, 2001). The function fitness evaluation included 30s chair-stand test, 30s one-arm curl test, chair sitand-reach test, back-scratch test, 2.44 meters up-and-go test, 2-min step test, and grips strength.Calculations were made using the Statistical Package for the Social Sciences(SPSS). All tests of significance adopted an alpha level of .05 .

\section{Results}

\subsection{Data of Participants}

This study recruited 25 participants. Older adults who did not have frailty syndrome, had an attendance rate that was lower than $70 \%$, or did not complete the entire test were excluded from the sample after the 12 weeks multicomponent training course. The final sample included 20 participants. Table 1 shows the results of the basic data survey. With regard to gender, most of the 11participants were female $(73.3 \%)$, while 4 participants $(26.7 \%)$ were male. The average age was $71.76 \pm 7.06$ years. The average height was $152.9 \pm 9.2 \mathrm{~cm}$. The average weight was $55.4 \pm 7.24 \mathrm{~kg}$.

Table1. Participants' basic data

\begin{tabular}{|c|c|c|c|c|}
\hline Gender & \multicolumn{2}{|c|}{ Number } & \multicolumn{2}{c|}{ Percentage } \\
\hline Male & \multicolumn{2}{|c|}{6} & \multicolumn{2}{c|}{$70.0 \%$} \\
\hline Female & \multicolumn{2}{|c|}{14} & Max. & Min. \\
\hline Variable & Mean & SD & 62 & 92 \\
\hline Age (years) & 79.77 & 7.06 & 47 & 140 \\
\hline Height $(\mathrm{cm})$ & 154.9 & 9.2 & 45 & 67.2 \\
\hline Weight $(\mathrm{kg})$ & 55.5 & 7.24 & \\
\hline
\end{tabular}




\subsection{Intervention on Cognitive Functions}

Table 2indicated that the results the total MMSE score was $18.27 \pm 3.327$ before the intervention and $22.93 \pm 3.945$ after the intervention. The average score increased from 17.07 before the intervention to 21.93 after the intervention, reaching a significant difference $(p=.001, p<.05)$. The results also showed that each part of cognitive function regarding changes in time orientation, spatial orientation, three-character word registration, attention and calculation, short-term memory, ability to recall names, repetition, understanding, reading, writing, drawing, and total MMSE scores after the multicomponent course intervention.

1) With regard to time orientation, its values were $2.70 \pm 1.468$ and $3.63 \pm 1.552$ before and after the intervention, respectively. The average values of the time orientation sense were 2.80 and $3.53 \mathrm{~d}$ before and after the intervention, respectively. However, the before and after results were reached significantly different $(p=.042, p<0.5)$.

2) The value for spatial orientation was $4.83 \pm .799$ before the intervention and $5 \pm .000$ after the intervention. Its average values were 4.73 and 5.00 before and after the intervention, respectively. However, the before and after results were not significantly different ( $p=.227, p>0.5)$.

3) With regard to the repetition of three-character words, the value was $2.97 \pm .352$ both before and after the intervention, and the average value did not change. The before and after results were not significantly different $(p=1, p>0.5)$.

4) The attention and calculation score was $.94 \pm 1.544$ points before the intervention and $1.53 \pm 1.959$ points after the intervention. The average scores were .93 and 1.53 before and after the intervention, respectively. However, the before and after results were not significantly different $(p=.238, p>0.5)$.

5) The short-term memory score was $.41 \pm .747$ before the intervention and $2.43 \pm 1.123$ after the intervention. The average scores were .40 and 2.43 before and after the intervention, respectively, reaching a significant difference $(p=.000, p<0.5)$.

6) With regard to understanding, the score both before and after the intervention was $.28 \pm .468$. The average score increased from 1.77 before the intervention to 2.80 after the intervention, reaching a significant difference $(p=.002, p<0.5)$.

7) The reading score was $.27 \pm .468$ before the intervention and $.27 \pm .468$ after the intervention., and the average reading score did not change. The before and after results were not significantly different $(p=1, p>0.5)$.

8) The writing score was $.08 \pm .278$ before the intervention and $.28 \pm .468$ after the intervention. The average score increased from .07 before the intervention to .27 after the intervention. However, the before and after results were not significantly different $(p=.082, p>0.5)$.

9) The drawing score before and after the intervention was $.34 \pm .498$ and $.48 \pm .526$, respectively. The average score increased from .33 before the intervention to .47 after the intervention. However, the before and after results were not significantly different $(p=.556, p>0.5)$.

10) The average scores for the ability to recall names and repetition did not change, and thus did not reach a statistically significant difference.

The statistical results indicated a significant change in the participants' MMSE total scores, time orientation, short-term memory, and understanding were reached a significantly different. However, in their scores on the before and after results including time orientation, spatial orientation, attention and calculation, writing, and drawing were not significantly different 
Effects of Multicomponent Exercise Prescription on Cognitive and Functional Fitness for Frailty Syndrome

Table2. Changes in MMSE scores

\begin{tabular}{|l|l|l|l|l|}
\hline Variable & Before Intervention & After Intervention & t-value & $p$-value \\
\hline Time orientation & $2.70 \pm 1.468$ & $3.63 \pm 1.552$ & -2.028 & $.042^{*}$ \\
\hline Spatial orientation & $4.83 \pm .799$ & $5.00 \pm .000$ & -1.293 & .227 \\
\hline Three-character word registration & $2.97 \pm .352$ & $2.97 \pm .362$ & .000 & 1 \\
\hline Attention and calculation & $.94 \pm 1.544$ & $1.63 \pm 1.969$ & -1.270 & .238 \\
\hline Short-term memory & $.40 \pm .747$ & $2.43 \pm 1.123$ & -6.808 & $.000^{* * *}$ \\
\hline Understanding & $1.67 \pm .986$ & $2.80 \pm .414$ & -3.900 & $.002^{* *}$ \\
\hline Reading & $.27 \pm .468$ & $.27 \pm .468$ & .000 & 1 \\
\hline Writing & $.08 \pm .278$ & $.28 \pm .468$ & -1.881 & .082 \\
\hline Drawing & $.34 \pm .498$ & $.48 \pm .526$ & -.619 & .546 \\
\hline Total & $18.27 \pm 3.327$ & $22.93 \pm 3.945$ & -4.172 & $.001 * *$ \\
\hline
\end{tabular}

\subsection{Intervention on Functional fitness}

The statistical results on Table 3 indicated a significant increase $(p<.001)$ in the participants' functional fitness scores. The result revealed that the functional physical fitness on shoulder flexibility and cardio respiratory endurance, lower muscles strengths, and mobility reached a significantly different.

Table3. Effects on functional fitness

\begin{tabular}{|c|c|c|c|c|c|}
\hline Fitness & Time & $\mathrm{M}$ & SD & $t$ & $p$ \\
\hline \multirow{2}{*}{$\begin{array}{l}\text { shoulder } \\
\text { flexibility }\end{array}$} & Before & -13.64 & 10.38 & \multirow[t]{2}{*}{$-5.49 * * *$} & \multirow[t]{2}{*}{.00} \\
\hline & After & -9.66 & 9.03 & & \\
\hline \multirow{2}{*}{$\begin{array}{l}\text { cardiorespiratory } \\
\text { endurance }\end{array}$} & Before & 101.26 & 7.29 & \multirow[t]{2}{*}{$-5.37 * * *$} & \multirow[t]{2}{*}{.00} \\
\hline & After & 145.45 & 6.63 & & \\
\hline \multirow[t]{2}{*}{ muscles strength } & Before & 15.70 & 2.87 & \multirow[t]{2}{*}{$-2.86 * * *$} & \multirow[t]{2}{*}{.00} \\
\hline & After & 17.53 & 2.66 & & \\
\hline \multirow[t]{2}{*}{ mobility } & Before & 10.21 & 1.91 & \multirow[t]{2}{*}{$3.99 * * *$} & \multirow[t]{2}{*}{.00} \\
\hline & After & 9.33 & 1.21 & & \\
\hline
\end{tabular}

$\mathrm{N}=15, * * * p<.001$

\section{DISCUSSION}

The purpose of this study was to examined the effects of a multicomponent exercise on cognitive function and functional fitness among the frail elderly. This study used multicomponent exercise prescription and applied a totally well instructional design model for frail elders. Twelve weeks of multicomponent exercise improved cognitive function in older adults with frailty syndrome. In particular, positive effects were participants' MMSE total scores, time orientation, short-term memory, and understanding were reached a significantly different. In addition, the result revealed that the functional physical fitness on shoulder flexibility and cardiorespiratory endurance, lower muscles strengths, and mobility reached a significantly increases.

Frailty is a geriatric syndrome that affects multiple domains of human functioning. A variety of problems contributes to the development of this syndrome; three key components includingsarcopenia, cognitive impairment, such as Alzheimer disease, and poor nutritional status were an important determinant of this condition (Heath, \& Stuart, 2002; Laura Lorenzo-López, et al., 2016; Sayer, et al, 2008).

The results of this study lend support to others who indicate that exercise prescription positively affects frail elders' cognitive and functional fitness (Erickson, et al.,2012; Suzuki, et al., 2017; Smith, et al. 2010).A retrospective review study revealed that aerobic exercises combined with double-task training or stretching exercises improved cognitive and motor functions in adults with MCI or early Alzheimer's disease (Chu, 2012). This study also is consistent with findings thatexercise improve attention, executive function, and memory and that both aerobic exercise and resistance training are important for the maintenance of cognitive and brain health in older adults (Smith et al., 2010; Voss et al., 2011; Wilson, Jackson, Sapey, \&Lord,2017). The results of this study was revealed that a multicomponent exercise may provide an effective way on cognitive function and functional fitness among the frail elderly. 
A more comprehensive design can improve all-round cognitive functions and fitness of frail elders and delay their degeneration(Lautenschlager, Cox, Flicke, et al, 2008).Thus, drawing from past research, this study developed amulticomponent exercise and totally well instructional design model of prescribing exercise for frail elders may improve the physical functions of older adults and thereby help to promote cognitive function on their frailty.

\section{CONCLUSiOn ANd SugGestions}

The issue of frailty syndrome is becoming increasingly important as the population of older adults grows. Frailty can also be seen as a biological syndrome that includes a reduced level of resistance in the face of multiple sources of pressure due to a decline in physiological and psychological functions. A multicomponent exercise program on performance effectively improves cognitive functions and promote the functional fitness. Furthermore, the results in this study indicated that twelve weeks of multicomponent exercise improved cognitive function on the MMSE total scoresamong older adults with frailty syndrome. In particular, positive effects weretime orientation, short-term memory, and understanding were reached a significantly different. In addition, the result revealed that the functional physical fitness on shoulder flexibility and cardio respiratory endurance, lower muscles strengths, and mobility reached a significantly increases. This study indicates that multicomponent exercise on dual-task performance can improve the functions fitness of older adults and thereby help to prevent or reduce their frailty.

In addition, the design of the course, a totally well instructional design model of prescribing exerciseas developed in this study for such patientswith frailty syndrome, included cognitive function training stimulating working memory, attention distribution, and the ability to plan. The result of this study indicated that the positive effects of the 12-week multicomponent training course improved the cognitive functions and fitness.

This study has some implications for those who design instruction for frail elders. The effects of thetotally well instructional design model of prescribing exercise on multicomponent training program can successfully increase frail elder performance and motivation. According the past study, some of the studieswere originally designed for one way's function among instructional settings. The current study suggests that the multicomponent training program can be used with instructional activities for frail elders. An application of designers should evaluate frail elder's characteristics, for example, ability, different age group with decline in physiological and psychological functions when assigning learners to instructional environment to accomplish goals.

Future studies should examine the relatively short duration of the treatment and the effect of instructional control may have influenced the outcomes. Extending the experimental time for overall instruction could produce different results for effective and performance. Further research should identify critical elements on cognitive function and fitness under which effective exercise prescription can be generated and maintained on the domain of functional training setting for frail elders. No control group was established in this study. Future studies should also establish a control group in order to observe its performance and analyze differences before and after multicomponent training. A future follow-up investigation is required to determine whether the effectexercise prescription is associated with prevention or evaluation on the stage of frailty syndrome. Additional research is needed to determine whether motivational factors may impact the learner's prior cognitive and frail stage to process training program effectively. For example, the participant's motivational states, attitudes about training program and self-esteem level may all play a part in their physical fitness or cognitive states to successfully interact with learning outcome.

\section{REFERENCES}

[1] American College of Sports Medicine. (2016). ACSM'S exercise for older adults. Philadelphia, USA.

[2] American College of Sports Medicine. (2009). Exercise and physical activity for older

[3] adults. Medicine and Science in Sports and Exercise, 41, 1510-1530.

[4] Angulo J, El Assar M, Rodríguez-Mañas L. (2016). Frailty and sarcopenia as the basis for the phenotypic manifestation of chronic diseases in older adults.Mol Aspects Med. 2016 Aug; 50:1-32. doi: 10.1016/j.mam.2016.06.001. Epub Jun 28.

[5] Bortz, W. M. (2002). A conceptual framework of frailty: A review Journal of Gerontol. A. Biol. Sci. 57: M283-8. 
Effects of Multicomponent Exercise Prescription on Cognitive and Functional Fitness for Frailty Syndrome

[6] Chu, Y. H. (2013). The effects of physical activity in cognitive function. The Journal of Long-term Care, 17(1), 11-21

[7] Erickson, K. I, Weinstein AM, Lopez O. L. (2012). Physical activity, brain plasticity, and Alzheimer's Disease.Arch Med., Res;43:615-21. Folstein, M. F., Folstein, S. E., \& McHugh, P. R. (1975). "Mini-mental state" : a practical method for grading the cognitive state of patients for the clinician. Journal of psychiatric research, 12(3), 189-198.

[8] Fried, L. P., Tangen, C. M., Walston, J., et al. (2001). Frailty in older adults: Evidence for a phenotype. Journal of GerontolBiol Med Sci., 56: M146-56.

[9] Heath, J. M. \& Stuart, M. R. (2002). Prescribing exercise for frail elders, JABFP, May-June, Vol. 15 No. 3.

[10] Huang, C. Y., Wang, H. H,\& Lin, M. J. (2016). The effects of functional physical and mental fitness platform for active ageing. Annual global report on Innovation in Active, Healthy and Smart Ageing Sector. Beijing Science \& Technology Publishing Press.

[11] Huang, C. Y., \& Wang, H. H. (2018). Prescribing Exercise for Frail Elders: A Cognicise Fitness Training Intervention as an Example. Annual global report on Innovation in Active, Healthy and Smart Ageing Sector. Beijing Science \& Technology Publishing Press.

[12] Jones, C. J., \& Rose, D. J. (2005). Physical activity instruction of older adult. ISBN: 07360-45139, Champaign, IL: Human Kinetics, P. O. Box 5076.

[13] Keevil V. L, Romero-Ortuno R. (2015). Ageing well: a review of sarcopenia and frailty. Proc Nutr Soc. 2015 Nov; 74(4):337-47. doi: 10.1017/S0029665115002037. May 25.

[14] Laura Lorenzo-López, Ana Maseda, Carmen de Labra, Laura Regueiro-Folgueira, José L. RodríguezVillamil, and José C. Millán-Calenti囚.( 2017). Nutritional determinants of frailty in older adults: A systematic review. Published online May 15.

[15] Lautenschlager, N. T, Cox, K. L, Flicker, L, et al. (2008). Effect of physical activity on cognitive function in older adults at risk for Alzheimer disease: a randomized trial. JAMA, 300:1027-37.

[16] Perracini, M. R., Franco, M. R. C., Ricci, N. A., \& Blake, C. (2017). Physical activity in older peopleCase studies of how to make change happen Best Practice \& Research Clinical Rheumatology,31 (2), 260274.

[17] Rikli, R. E., \& Jones, C. J. (2001). Senior fitness test. Champaign (IL) : Human Kinetics, 53(2),255-67.

[18] Ruan. Q.Yu, Z.Chen. M, Bao Z.Li. J.\&He. W. (2015). Cognitive frailty, a novel target for the prevention of elderly dependency.Ageing Res Rev. Mar;20:1-10.

[19] Sayer A. A, Syddall H, Martin H, Patel H, Baylis D, Cooper C. (2008). The developmental origins of sarcopenia.J Nutr Health Aging. Aug-Sep;12(7):427-32.

[20] Smith, P. J, Blumenthal J. A, Hoffman, B. M, Cooper H, Strauman, T. A, Welsh-Bohmer, K, Browndyke, J. N, Sherwood, A.( 2010). Aerobic exercise and neurocognitive performance: a meta-analytic review of randomized controlled trials. Psychosom Med.; 72(3):239-252. doi: 10.1097/PSY.0b013e3181d14633.

[21] Suzuki, T., , Shimada, H., Makizako, H., Doi, T., Yoshida, D., Tsutsumimoto, K., Anan, Y., Uemura, K., Lee, S., and Park, H. (2017). Community-Based Intervention for Prevention of Dementia in Japan,The Journal of Prevention of Alzheimer's Disease - JPAD. Volume 2, Number ,1-6.

[22] Voss, M. W., Nagamatsu, L. S., Liu-Ambrose, T., \& Kramer, A. F. (2011) . Exercise, brain, and cognition across the life span. Journal of applied physiology, 111(5), 1505-1513.doi: 10.1152/japplphysiol. 00210.2011 .

[23] Wilson D, Jackson T, Sapey E, Lord J. M. (2017). Frailty and sarcopenia: The potential role of an aged immune system. Ageing Res Rev. Jul; 36:1-10.

[24] Wojtek, J., \&Chodzko-Zajko, (2015). ACSM's Exercise for older adults. Kluwer Health, Philadelphia.

[25] Zhang, N, Zhu, W. L., Chen, W., Han, Y. C. (2019). A cross-sectional study on the association between frailty and muscular performance in hospitalized elder patients with coronary artery disease. Zhonghuaneizazhi, 58 (4), 265-269.

\section{AUTHORS' BIOGRAPHY}

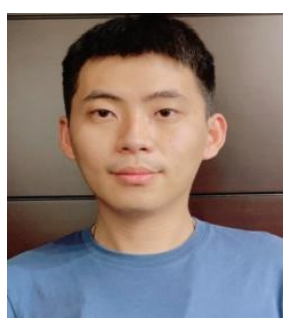

Pin-YuWu, is a candidate student of Ph. D program in Department of Adult and Continuing Education, National Chung-Cheng University, Chiayi, Taiwan, Taiwan, R. O. C.Wu's field of expertise lies in sports, health and recreation management, physical activity instruction of older adults, and the design of sport management for active-aging learners. 


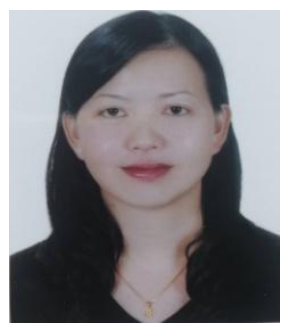

Lee-Lan Cheng, is a candidate student of $\mathrm{Ph}$. D program in Department of Adult and Continuing Education, National Chung-Cheng University, Chiayi, Taiwan, Taiwan, R. O. C. Currently, teaches physical education and functional fitness for older adults in society and is also anexternal evaluator for the fitness and consultant at a fitness promotion for society senior citizens. Cheng's field of expertise lies in sports and health promotion, sports pedagogy, Physical activity instruction of older adults, and the design of educational sports programs for active-aging learners.

Citation: Pin-Yu, Wu, Lee-Lan Cheng. " Effects of Multicomponent Exercise Prescription on Cognitive and Functional Fitness for Frailty Syndrome” International Journal of Humanities Social Sciences and Education (IJHSSE), vol 8, no. 5, 2021, pp. 41-48. doi: https://doi.org/10.20431/2349-0381.0805006.

Copyright: () 2021 Authors. This is an open-access article distributed under the terms of the Creative Commons Attribution License, which permits unrestricted use, distribution, and reproduction in any medium, provided the original author and source are credited. 